\title{
A reusable e-learning tool for paediatric physio- therapy education: a preliminary report
}

\section{Sarah Westwater-Wood ${ }^{1}$ and Reg Dennick ${ }^{2}$}

${ }^{1}$ Division of Physiotherapy Education, The University of Nottingham, UK

${ }^{2}$ Medical Education Unit, The University of Nottingham, UK

Correspondence: Sarah Westwater-Wood, Division of Physiotherapy Education, Clinical Sciences Building, Nottingham City Hospital Campus, Hucknall Road, UK. Email: sarah.westwater-wood@nottingham.ac.uk

Accepted: August 30, 2011

\begin{abstract}
Objectives: The aim of this study was to devise, implement and evaluate a paediatric contextualised reusable computeraided learning resource.

Methods: This study is an evaluation of a curriculum development in which all 21 third year undergraduate physiotherapists at the University of Nottingham participated. Data collection was via an online survey tool comprising open and closed questions using Likert scaling (1-5). Closed questions were aimed at ease of use, task setting and development of clinical reasoning skills. Two free text opportunities were provided.
\end{abstract}

Results: The five point Likert ranking most frequently selected was agree or strongly agree. The first prompt for "specific issues with practical use" provided a main theme of poor experience using podcast elements; whilst the second prompt "anything else ... you would like to add" provided a main theme of appreciation for the realism and problem solving skills stimulation.

Conclusions: The computer-aided learning resource was found to be an appropriate teaching and learning approach. Students perceived that it facilitated their practice of clinical case management with this patient group.

Keywords: Education, e-learning, computer-aided learning, physiotherapy, paediatrics

\section{Introduction}

Educational approaches for healthcare (HC) professionals need to stimulate problem-solving skills essential to professional practice. ${ }^{1}$ In paediatric physiotherapy there is an expectation of autonomous working where individual practitioners diagnose, prescribe treatment plans, refer onwards and follow up patients. Physiotherapists assess and diagnose in order to identify appropriate therapeutic interventions, which in the main involve exercise and mobilisations with various levels of movement advice. This extra 'movement' dimension is an organic process in physiotherapy practice ${ }^{2}$ and is a distinguishing feature of physiotherapists in comparison to other healthcare practitioners. ${ }^{3,4}$ In this complex area on-going problem solving and clinical reasoning development should be at the core of any educational program. Jenson et $\mathrm{al}^{1}$ describe a current state where the educational approaches to physical therapy practice within universities is disconnected from clinical environments. They advocate that teaching approaches and curriculum development should strive to bridge this gap. Within the academic environment real patient interaction and case management, which would stimulate these skills, is problematic as the patient group comprises children and their families. Children are a vulnerable patient group with many aspects of their assessment and treatment being mediated via parents/careers.

To bring representative children into the academic environment, even for small groups of students, would be unethical. The challenge is to find ways to solve this educational problem in the context of a paediatric care module set within undergraduate physiotherapy education. The chosen solution was to devise, build and implement a contextualised reusable computer-aided learning (CAL) programme which would be used as a stimulus to facilitate student learning. Over time this bespoke CAL program became known as the ReTool which was used in weekly, task-based, small group teaching sessions. The rationale for this pro- 
gramme combines research developments from the field of CAL as well as the use of a number of pedagogical frameworks described below. There is a growing body of literature outlining and evaluating the introduction of CAL within HC education across disciplines. ${ }^{5-15}$ Between 2001 and 2006 the proliferation of eLearning went from $11 \%$ to $29 \%$ in US training programs. ${ }^{16}$ The majority of CAL studies in HC have involved medical students as opposed to allied healthcare professionals. The main analytical approach in the published literature has been descriptive and inductive. There is less deductive evidence and no studies have been identified within the discipline of physiotherapy. Those studies which used surveying techniques describe positive accounts of CAL implementation. ${ }^{6,10-12,14,17}$

However, the quantitative researcher will consider this a significant weakness in methodological design ${ }^{9}$ since individual participants may have experienced non-CAL teaching and learning approaches which may have influenced their responses to qualitative survey techniques. In addition some studies have considered qualitative results alongside pre-existing assessments of learning, which have demonstrated congruence with qualitative findings. ${ }^{14}$ Four comparative studies addressed CAL for medical courses ${ }^{8,13}$, one orthodontic ${ }^{15}$ and one nursing. ${ }^{5}$ One study found that CAL taught groups proposed a wider range of relevant clinical factors in their care plan when compared to a nonCAL group. ${ }^{5}$ The authors suggested this was as a result of CAL stimulating deeper thinking. In another study comparison between individual students ${ }^{13}$ utilising a CAL program as an adjunct to existing traditionally taught programs used entry and exit exam ranking as an outcome measure. This revealed a positive result for the use of CAL and an improvement in ranking. ${ }^{8,13}$ Velan et al ${ }^{8}$ considered a 3 rd and 4 th year medical pathology module where students volunteered to take part in a two group intervention and nonintervention study design. The main finding was statistically significant higher test results in the CAL group compared to the non-CAL group. In the discussion the authors reported positive attitudes to the CAL from focus group findings. Additionally CAL study outcomes have shown that visual imagery, choice and usability are identified with positive outcomes for CAL projects. ${ }^{8,10,15}$

Barriers to successful implementation of CAL, which studies identified, included timetabled or ring fenced time and costs. ${ }^{9,12}$ Only one study identified CAL as more acceptable to students than traditional teaching ${ }^{14}$ with one review finding it equal overall. ${ }^{18}$ A number of authors cautioned against the use of CAL as a substitute for tutor/lecturer time. ${ }^{7,9}$ There is evidence that in designing instructional materials student perceptions will be dominated by accessibility in terms of visuals and ease of engagement whereas teacher-designers are more likely to consider pedagogical aspects. ${ }^{19}$ Thus novices apparently want different learning materials than the expert who is designing the instructional package. Indeed a number of studies have identified visual components as key for learners. 6, 17, 18,20

Instructional texts for CAL developers highlight the broader evidence of visual and audio prominence, along with limiting text as dominating factors for success with CAL learning packages. ${ }^{16,20,21}$ Closely related to the use of both near and far transfer concepts in CAL development is the coherence principle. The coherence principle dictates that adding anything not specifically supporting the learning objectives should be avoided. ${ }^{16}$ Indeed when utilising both visuals and text CAL is most successful when biased towards its strength in multimedia formats. ${ }^{16,22}$ However, a cautionary note derives from the cognitive theory of learning which suggests that as text and visuals both require the same visual pathways, when presented simultaneously, conflict in processing can occur. ${ }^{16,20}$ Consequently, for the educator who is a novice in the production of CAL packages, there are many potential barriers to success during the design phase (of which only a few key factors have been considered here).

There are other factors which also need to be taken into consideration when developing educational packages for clinical professionals. An earlier view of the development of professional expertise, which has informed approaches to teaching ${ }^{22-24}$ is that the acquisition of extensive knowledge is followed by its application in response to patient problems. However, more recent research findings support the view that expert knowledge and its application develop together. ${ }^{24-26}$ Thus the evolution of approaches to expert education highlight the importance of learning and facilitation by clinically contextualised tasks set in the zone of proximal development (zpd) as defined by Vygotsky. ${ }^{27}$ Both expert and peer facilitation ${ }^{26,28}$ and the opportunity to practice authentic activities ${ }^{23,24}$ are positive contributions to novicepractitioner development and are a major feature of the development described here.

In addition there are identifiable processes in the ways in which experts mentor and teach novices which can be incorporated into facilitation technique. Renninger and Winegar ${ }^{29}$ for example presented a structure in which the expert paces information for the novice so as to facilitate them "owning" the knowledge desired. Although the emphasis is upon knowledge the framework also holds true for critical thinking and knowledge application. We might term this the 'art' of teaching, where the organisation and pacing of information and the developing independence from the expert, is assessed by the expert in real time as they become aware of the novices growing abilities. ${ }^{26,28,29}$ Thus, the expert progressively reduces 'constraints' on the novice in carrying out a task.

In conclusion, although the use of CAL in $\mathrm{HC}$ education has been positively reported in a variety of medical disciplines, no studies of CAL in physiotherapy education or paediatric practitioner disciplines have been undertaken. 
The ReTool study reported here describes the implementation and evaluation of a CAL package, linked to small group facilitation and based on sound instructional and pedagogical principles, providing authentic paediatric case studies.

\section{Methods}

\section{Study participants, CAL development, and implemen-} tation

As this study was a curriculum development and evaluation in an academic environment no ethical approval was required. Participants were 21 undergraduate physiotherapy students in their third year of study. In order to create the reusable e-Learning tool for paediatric case study management an electronic template was constructed. This was named the ReTool for ease of project communications. The ReTool was mounted on the University of Nottingham's existing virtual learning environment, WebCT. For the pilot a single contextualised case study was developed using multimedia and web links to populate the ReTool. Life-like documents and resources were used to enhance realism.

\section{Use of the ReTool}

The ReTool was used as an educational resource over a four week period of the undergraduate Paediatric Care Module (PCM). At each weekly session the ReTool presents a group of 4-5 students with a task, for example to plan an objective assessment. In order to facilitate their thinking they are presented with a choice of items exactly as a clinician would encounter in real clinical practice and in a range of appropriate formats. For example there are Word documents of referral letters and written reports from healthcare professionals who would be involved with the case. Other items such as a history taking interview with parents or telephone conversations with other non-healthcare agencies use audio-video and audio formats.

A typical sequence is outlined below:

Week 1: Developing patient specific history taking skills. For example planning a history taking consultation with parent and child.

Week 2: Seeing the actual history taking, reflecting on what the students had planned and what they had omitted. Developing an objective assessment.

Week 3: Seeing the actual objective assessment. Planning treatment options.

Week 4: Focusing upon treatment options and the natural course of pathology. Writing a letter back to the referrer.

\section{Data Collection}

\section{Questionnaire}

Students were surveyed for their views of the ReTool implementation by means of an online questionnaire based upon a previously published instrument..$^{30-32}$ It comprised a mixture of open and closed questions using a Likert scale ( 1 = strongly agree, 2 = agree, 3 = acceptable, $4=$ disagree, 5 = strongly disagree). Principle areas addressed were ease of use, teaching and learning approach and whether learning outcomes were met. There were two opportunities provided in the questionnaire for free text comments.

The first prompt was "Please use the space below to comment on any specific issues with practical use of the ReTool". The second occurred at the conclusion of the questionnaire where a free text box was provided with the prompt "Please use the space below to say anything else about the ReTool which you would like to add". The questionnaire was administered using an online programme, the link for which was emailed to students in the following week after completion of the module. Within this small scale evaluation rigorous analysis of the questionnaire for validity and reliability was not possible. ${ }^{33}$ However, basic face validity was considered after several discussions with colleagues and supported in so far as the analysis allowed the domain under consideration to be evaluated using a 5 point Likert scale. ${ }^{34}$

\section{PCM Summative assessment}

The PCM summative assessment consisted of one option from a choice of 3 titles. To assess the ReTool students were provided with one option based on a referral letter concerning their case study and including a 1000 word essay regarding the pathology and current evidence base for the physiotherapeutic approaches to its management. To avoid disadvantaging any student, and for comparative purposes, students were also given the choice of two conventional 3000 word essay titles concerning paediatric physiotherapy/pathology. Analysis of these summative assessment results provided quantitative data for evaluating the effectiveness of the ReTool approach.

\section{Analysis of Data}

The questionnaire data were processed in Excel to produce percentages. The open responses were analysed for themes. The summative assessments were marked by standardised marking criteria using a percentage scale. Marking was blinded by use of personal identification numbers rather than student names and all scripts were second marked.

\section{Results}

Of the 21 students who undertook the module 16 (14 female, 2 male) took part in the survey (76\% response rate). However 2 respondents did not fully complete all components of the questionnaires hence the final response rate was $66.6 \%(\mathrm{~N}=14)$.

\section{Questionnaire results}

ReTool implementation was rated highly by students for achieving the module learning outcomes for problem solving and clinical reasoning skills (Table 1). 
Table 1. Results of Likert ranking (\%)

\begin{tabular}{|c|c|c|c|c|c|}
\hline Level of agreement for ReTool learning outcomes & $\begin{array}{l}\text { Strongly } \\
\text { Disagree }\end{array}$ & Disagree & Acceptable & Agree & $\begin{array}{c}\text { Strongly } \\
\text { Agree }\end{array}$ \\
\hline To develop knowledge and understanding of a specific paediatric pathology & & 7 & 7 & 50 & 29 \\
\hline To develop clinically provoked PS skills and confidence & & 7 & 14 & 36 & 43 \\
\hline To develop CR in regard to management of a paediatric condition & & & 14 & 43 & 43 \\
\hline To deepen knowledge and understanding of physiotherapist's role & & 7 & & 64 & 29 \\
\hline \multicolumn{6}{|l|}{ Level of agreement for ReTool ease of use } \\
\hline Overall how easy was the ReTool to use? & & & 29 & 57 & 14 \\
\hline How easy were the videos to use? & & & 36 & 50 & 14 \\
\hline How easy were the audio to use? & 7 & 28 & 29 & 22 & 14 \\
\hline How easy were the web-links to use? & & & 43 & 43 & 14 \\
\hline How easy were the documents to use? & & & & 43 & 57 \\
\hline
\end{tabular}

Level of agreement for ReTool facilitation and task setting levels

Was the task set at the right level?

$\begin{array}{lll}21 & 50 & 27 \\ 14 & 43 & 43 \\ 14 & 50 & 36\end{array}$

Were the facilitation sessions useful?

Were the facilitation sessions set at the right level?

Students agreed that it was easy to use and rated the audio content (podcast) as acceptable. Student views on the level and use of facilitation sessions and the level of tasks set also demonstrated ratings towards agreement and strong agreement.

\section{Themes derived from open questions}

Altogether there were 19 submitted responses, 12 in reply to the first prompt and 7 to the second, generating four themes. The results of the thematic analysis are shown in Table 2.

Table 2. Themes arising from open questions $(n=14)$

\begin{tabular}{ll}
\hline First Prompt & Number of times mentioned \\
\hline Theme & - \\
Quality of audio & 7 \\
Getting together for group work & \\
\hline Second Prompt & - \\
\hline Theme & 5 \\
Realism and overt problem solving & 2 \\
Caution & \\
\hline
\end{tabular}

The first prompt provided two key themes. One reveals a perceived poor experience in using the audio (podcast) elements of the ReTool for example:

"Sometimes the audio was too quiet, even with speakers attached" - Third year student

And:
"The audio was not very clear and not loud enough" - Third year student

The other theme provides insight into the difficulties perceived by the small groups in timetabling sessions to meet. Examples of issues submitted within this theme are as follows:

"Group discussions were difficult with ReTool as group mates had different modules and were in on different days" - Third year student

And:

"Main limitation was in finding practical times in which the whole group could meet” - Third year student

The second prompt provided one clear main theme plus one other minor theme. The main theme provided a perceived appreciation of the realism and understanding that problem solving skills had been stimulated, whilst the other theme was one of perceived caution. Examples of comments, which had the theme of 'realism' and appreciation of active problem solving, are as follows;

“. . feel if I'd had the ReTool regarding basic musculoskeletal injuries in first year.. it would greatly have improved my problem solving and relation to the rest of the course at an earlier date" - Third year student

And:

"Made learning about the pathology interesting, challenging and more realistic" 


\section{Coursework marks}

The range of marks for the PCM summative assessments, taken by all 21 students, are presented in Table 3 demonstrating that the median and maximum mark for each item of coursework are within 4 marks of each other. The minimum mark is marginally higher for those selecting the respiratory essay title whereas the cerebral palsy and ReTool option minimum marks are within two marks of each other. When comparing those students on the PCM who chose the assessment title linked to the ReTool as compared to those who chose the established essay format (Table 3), there was no trend to skew marks towards one particular item of coursework.

\section{Discussion}

The primary aim of the study was achieved with the development and implementation of a reusable computer-aided learning tool. Student evaluation of the ReTool implementation demonstrated a successful teaching and learning development within paediatric physiotherapy. Surveyed students responded that their clinical reasoning and problem-solving skills were stimulated and developed by engagement with the ReTool. This was also supported by the facilitator's informal reflections on the facilitation sessions and is compatible with the wider adoption of CAL use in HC professional education. ${ }^{3-13}$

Table 3. PCM summative assessment marks $(n=21)$

\begin{tabular}{lccc}
\hline & $\begin{array}{c}\text { Essay 1 }\left(\mathrm{CP}^{*}\right) \% \\
(\mathrm{n}=7)\end{array}$ & $\begin{array}{c}\text { Essay 2 }\left(\text { Resp }^{\dagger}\right) \% \\
(\mathrm{n}=3)\end{array}$ & $\begin{array}{c}\text { ReTool }^{\ddagger} \% \\
(\mathrm{n}=11)\end{array}$ \\
\hline Minimum & 48 & 67 & 50 \\
Maximum & 84 & 80 & 82 \\
Median & 69 & 69 & 64 \\
\hline
\end{tabular}

* Essay title, "In a child with diplegic cerebral palsy consider the most common gait * Essay title, "In a child with diplegic cerebral palsy consider the most common gait presentation in the light of the above statement. What is the
'frequency' with which the secondary abnormalities can be corrected.

trequency" with which the secondary abnormalities can be corrected. tessay tile, "Discuss the PT role in the management of children with chronic respiratory disease specifically focusing upon outcome measures a physiotherapist would utilise to ensure 'best care of a changing' disorder and that nothing is overlooked.

Y You are the physiotherapist for your case study. Write a letter to the referrer with the report. You should submit a short, evidenced, essay which describes the epidemiology, pathology, treatment approaches and role of the physiotherapist as related to the case study.

Students recommended the ReTool for on-going paediatric and wider educational applications. However, as has been found in other studies ${ }^{7,9}$ there was a small voice of caution against CAL dominating as an educational approach (Table 2). This is appropriate for a practical skill based profession such as physiotherapy. Also, as with prior reports, participating students found timetabling for group working difficult. ${ }^{7}, 10$

With regard to far transfer within the paediatric physiotherapy case management role there are many opportunities for layering learning. A particular feature of the ReTool design which addressed this was the week-by-week choices selected by the students. Specifically all the choices offered each week mimicked the 'real' life situation where there are different routes to capture pertinent information about the patient and the case. Limiting the use of text items, with an emphasis on video and audio, appears to have been successful in addressing coherence and reducing cognitive conflict for the ReTool users.

Renninger and Winegar ${ }^{29}$ discuss the influence of learning style upon learning environment and task construction, citing Montessori's work on facilitating environments rather than specific tasks. ${ }^{29}$ This has similarities to wanting HC students within the academic environment to have contextualised clinical case studies to manage which stimulate problem identification, solution finding, decision making, justification and evaluation skills. The objective in this study was to partially bridge the gap between the academic and clinical environment as identified by Jenson et al. ${ }^{1}$ The question which arises then is whether the ReTool can act as a stimulating presenter of case studies? Whilst we need to be cautious, due to the small sample size, the findings of this preliminary study, including the positive open comments and the high ratings given to the ReTool as a stimulus to clinical reasoning and problem-solving skills, suggests it did provide a stimulating learning environment.

This study has a number of limitations. It was a preliminary study from a single institution using a survey approach with a small module intake and thus the sample size is small. A further limitation is the lack of an objective outcome measure for problem solving and clinical reasoning skills. Future testing of outcomes might involve the use of reflective practice diaries, objective structured practical assessments or the use of script concordance testing to test clinical reasoning skills. ${ }^{35}$

\section{Conclusions}

Currently there is consensus within the literature that CAL use should be 'in the mix' in terms of modalities for the learning exchange rather than as a replacement for lecturer based teaching approaches. This is compatible with the evolution of educational approaches which emphasise expert input in both the construction of tasks and provision of scaffolding to facilitate student learning. Therefore, in addressing the learning objectives for the PCM, to facilitate novice student physiotherapists in their practice of clinical case management, the development of the ReTool CAL package was found to be an appropriate teaching and learning approach to pilot. Subsequent to the pilot study three more case studies have been developed to populate the wireframe. These have been implemented within undergraduate physiotherapy education at the University of Nottingham.

These preliminary findings support the use of this CAL tool for paediatric physiotherapy education. In undergraduate studies there is potential to consider application to interprofessional learning. In post graduate development those new or returning to clinical paediatric practice may find utilisation of this or similar CAL tools of benefit. More broadly discussion around the case studies and decisions 
may promote sharing of good practice both uni professionally and inter-professionally. However, implementation of CAL should be guided by its own strengths in facilitating teaching and learning, supported by wider educational philosophies and hence should not be used simply for its own sake.

\section{Acknowledgements}

During the development of the case studies contributions were made by patients and carers and a wide range of allied health professionals whose contributions were invaluable.

\section{Conflict of Interest}

The authors declare that they have no conflict of interest.

\section{References}

1. Jenson G, Gwyer J, Hack L, Shepard K. Expertise in physical pherapy practice. 2nd ed. Missouri: Saunders; 2007.

2. James G. Modelling diagnosis in physical therapy: a blackboard framework and models of experts and novices. Ergonomics. 2007;50(3):335-51.

3. Shepard K, Hack L, Gwyer J, Jenson G. Describing expert practice in physical therapy. Qualitative Health Research. 1999;(9):746-58.

4. Doody C, McAteer M. Clinical reasoning of expert and novice physiotherapists in an outpatient orthopaedic setting. Physiotherapy. 2002;88:258-68.

5. Gwinnett A, Massie S. Integrating computers into the curriculum (2): transforming a concept into a working tool. Nurse Education Today. 1987;(7):116-9.

6. Lowery M, Johnson M. Computer assisted learning: the potential for teaching and assessing in nursing. Nurse Education Today. 1999;(19):521-6.

7. Steele D, Johnson Palensky J, Lynch T, Lacy N, Duffy S. Learning preferences, computer attitudes, and student evaluation of computerised instruction. Medical Education. 2002;(36):225-32.

8. Velan G, Killen MT, Dziegielewski M, Kumar RK. Development and evaluation of a computer-assisted learning module on glomerulonephritis for medical students. Medical Teacher. 2002;24(4):412-6.

9. Vogel M, Wood D. Love it or hate it? Medical students' attitudes to computer-assisted learning. Medical Education. 2002;(36):214-5.

10. Denny M, Higgins A. The use of computer assisted technology to enhance student psychiatric nurses learning during a practice placement. Nurse Education in Practice. 2003;(3):80-8.

11. Sturmberg J, Crowe P, Hughes C. Computer-assisted instruction: guiding learning through a key features approach in a community-based education course. Medical Teacher. 2003;25(3):332-5.
12. Shaw S, Kerr R, Carter YH, Leighton-Beck L, Farnham $\mathrm{M}$. Evaluation of an interactive educational tool for primary care researchers. Medical Teacher. 2004;26(5):483- 6.

13. Healy D, Fleming FJ, Gilhooley D, Felle P, Wood AE, Gorey $\mathrm{T}$, et al. Electronic learning can facilitate student performance in undergraduate surgical education: a prospective observational study. BMC Medical Education. 2005;5:23.

14. Zebrack J, Mitchell JL, Davids SL, Simpson E. Webbased curriculum; a practical and effective strategy for teaching women's health. J GEN INTERN MED. 2005;(20):68-74.

15. Aly M, Willems G, Carels C, Elen J. Instructional multimedia programs for self-directed learning in undergraduate and postgraduate training in orthodontics. European Journal of Dental Education. 2003;(7):20-6.

16. Clark R, Mayer R. E-Learning and science of instruction. 2nd ed. San Francisco: Pfeiffer; 2008.

17. Vikram J, Widdowson S, Duffy S. Development and evaluation of an interactive computer-assisted learning program-a novel approach to teaching gynaecological surgery. British Journal of Educational Technology. 2002;33(3):323-31.

18. Tallent-Runnels M, Thomas J, Lan W, Cooper S. Teaching courses online: a review of the research. Review of Educational Research. 2006;76(1):93-135.

19. Price L. Lecturers' vs. students' perspectives of the accessibility of instructional materials. Instructional Science. 2007;(35):317-41.

20. Mayer R, Moreno R. Nine ways to reduce cognitive load in multimedia learning. Educational Psychologist. 2003;38(1):43-52.

21. Lopuck L. Web design for dummies. 2nd ed. New York: Wiley Publishing Inc; 2006.

22. Higgs J, Jones M, Loftus S, Christensen N. Clinical reasoning in the health professions. 3rd ed. UK: Butterworth Heinmann Elsevier; 2008.

23. Benner P, Tanner C. How expert nurses use intuition. American Journal of Nursing. 1987;(1):23-31.

24. Schon D. The reflective practitioner: how professionals think in action. Aldershot, England: Ashgate Publishing Ltd; 1991.

25. Higgs J, Titchen A. Research and knowledge. Physiotherapy. 1998;84(2):72-80.

26. Gillespie M. Equipped for the future research principle: an approach to teaching and learning that builds expertise. Information analyses RFF Research to Practice Note 2. 2002;(070):1-8.

27. Daniels H. An introduction to Vygotsky. New York: Rutledge; 1996.

28. Spouse J. Bridging theory and practice in the supervisory relationship: a sociocultural perspective. Issues and Innovations in Nursing Education. 2001;33(4):512-22. 
29. Renninger K, Winegar L, editors. Organization of teacher-student interaction: differential constraining progressive empowerment. The Annual Convention of the American Psychological Association (93rd) 1985 August 23-27; Los Angeles, CA: American Psychology Association. 30. Zebrack JR, Mitchell JL, Davids SL, Simpson DE. Webbased curriculum. A practical and effective strategy for teaching women's health. Journal of General Internal Medicine. 2005;20:68-74.

31. Velan GM, Killen MT, Dziegielewski M, Kumar RK. Development and evaluation of a computer-assisted learning module on glomerulonephritis for medical students. Medical Teacher. 2002;24(4):412-6.
32. Albaum G. The Likert scale revisited: an alternate version (product preference testing). Journal of the Market Research Society. 1997;39(2):1-12.

33. Rattray J, Jones M. Essential elements of questionnaire design and development. Journal of Clinical Nursing. 2005; 16:234-43.

34. Thomas JR, Nelson JK. Research methods in physical activity. 4th ed. Australia: Human Kinetics; 2001.

35. Charlin B, Brailovsky C, Roy L, Goulet F, van der Vleuten C. The script concordance test: a tool to assess the reflective clinician. Teaching and Learning in Medicine. 2000;12:189-195. 